\title{
VIRTUALLY GEOMETRIC WORDS AND WHITEHEAD'S ALGORITHM
}

\author{
JASON FOX MANNING
}

\begin{abstract}
Motivated by a question of Gordon and Wilton, we consider the question of which collections of words are "virtually geometric". In particular, we prove that some words (e.g. bbaaccabc) are not virtually geometric.
\end{abstract}

\section{Definitions and introduction}

Let $G$ be a group, $g=\left\{\left[g_{i}\right]\right\}_{i \in I}$ a collection of conjugacy classes in $G$, and $X$ some space. Any homomorphism $\phi: G \rightarrow \pi_{1}(X)$ induces a map,

$$
\phi_{\underline{g}}: \bigsqcup_{i \in I} S^{1}{ }_{i} \rightarrow X
$$

well-defined up to homotopy, which sends the circle $S^{1}{ }_{i}$ to a loop in $X$ freely homotopic to the element $\phi\left(g_{i}\right)$.

Definition 1.1. Let $F_{n}=\left\langle x_{1}, \ldots, x_{n}\right\rangle$ be the free group of rank $n$, and let $H$ be a 3-dimensional handlebody of genus $n$. We say a collection of conjugacy classes $\underline{w}=\left\{\left[w_{1}\right], \ldots,\left[w_{r}\right]\right\}$ in $F$ is geometric if for some isomorphism

$$
\phi: F_{n} \rightarrow \pi_{1}(H)
$$

the induced map $\phi_{\underline{w}}$ is homotopic in $H$ to an embedding

$$
\phi_{\underline{w}}^{\prime}: \bigsqcup_{i=1}^{r} S^{1}{ }_{i} \hookrightarrow \partial H .
$$

In this case we also say that the finite presentation

$$
\left\langle x_{1}, \ldots, x_{n} \mid w_{1}, \ldots w_{r}\right\rangle
$$

is geometric.

Remark 1.2. Let $\phi: F_{n} \rightarrow \pi_{1}(H)$ be an isomorphism as in Definition 1.1. Since any automorphism of a free group $F_{n}$ can be realized as a homeomorphism of the handlebody $H$, the phrase "for some isomorphism" can be replaced by "for any isomorphism". (To see that any automorphism can be realized, first realize elementary Nielsen transformations

$$
\alpha_{i}: \quad x_{i} \mapsto x_{i}^{-1}, x_{j} \mapsto x_{j}, \forall j \neq i,
$$

and

$$
\beta_{i j}: \quad x_{i} \mapsto x_{i} x_{j}, x_{k} \mapsto x_{k}, \forall k \neq i,
$$

and then use the fact that these transformations generate $\operatorname{Aut}\left(F_{n}\right)$, [13, I.4].)

Received by the editors April 18, 2009. 
Remark 1.3. A geometric presentation $\left\langle x_{1}, \ldots, x_{n} \mid w_{1}, \ldots w_{r}\right\rangle$ gives a (not necessarily unique) way to build a 3 -manifold $M$ with $\pi_{1}(M)=\left\langle x_{1}, \ldots, x_{n} \mid w_{1}, \ldots w_{r}\right\rangle$, by attaching 2 -handles to the boundary of the handlebody $H$, along attaching curves $\phi_{w}^{\prime}\left(S^{1}{ }_{i}\right)$ for $1 \leq i \leq r$. Thus every geometric presentation is a presentation of the fundamental group of some 3 -manifold.

Moreover, the double $D_{n}(\underline{w})$

$$
\left\langle\begin{array}{c|c}
x_{1}, \ldots, x_{n}, & w_{1}\left(x_{1}, \ldots, x_{n}\right)=w_{1}\left(x_{1}^{\prime}, \ldots, x_{n}^{\prime}\right), \\
x_{1}^{\prime}, \ldots, x_{n}^{\prime}, & t_{i}\left(w_{i}\left(x_{1}, \ldots, x_{n}\right)\right) t_{i}^{-1}=w_{i}\left(x_{1}^{\prime}, \ldots, x_{n}^{\prime}\right), i \geq 2
\end{array}\right\rangle
$$

is the fundamental group of a 3-manifold built by attaching thickened annuli to two copies of the handlebody $H$ of genus $n$, along the attaching curves given by $\phi_{\underline{w}}^{\prime}$.

Definition 1.4. Let $\underline{w}=\left\{\left[w_{1}\right], \ldots,\left[w_{r}\right]\right\}$ be a collection of conjugacy classes in the free group $F=F_{n}$. For $F^{\prime}<F$ a finite index subgroup, we define the lifts of $\underline{w}$ to be the following set of $F^{\prime}$-conjugacy classes:

$$
\underline{w}_{F^{\prime}}=\left\{\left[g w^{n(g)} g^{-1}\right] \mid \quad n(g) \in \mathbb{Z}_{>0} \begin{array}{l}
g \in F, w \in \underline{w}, \text { and } \\
\text { minimal so that } g w^{n(g)} g^{-1} \in F^{\prime}
\end{array}\right\}
$$

The collection $\underline{w}$ is virtually geometric if $\underline{w}_{F^{\prime}}$ is geometric for some finite index $F^{\prime}<F$. In this case, we say the presentation

$$
\left\langle x_{1}, \ldots, x_{n} \mid w_{1}, \ldots w_{r}\right\rangle
$$

is virtually geometric.

Here is an equivalent topological formulation: Realize $\underline{w}$ as an embedded collection of circles $N_{\underline{w}}$ in $H$. If, for some finite cover $\tilde{H} \stackrel{p}{\rightarrow} H$, the inclusion

$$
\tilde{\iota}: p^{-1}\left(N_{\underline{w}}\right) \rightarrow \tilde{H}
$$

is homotopic to an embedding into $\partial \tilde{H}$, then $\underline{w}$ is virtually geometric.

Remark 1.5. If a presentation $\left\langle x_{1}, \ldots, x_{n} \mid w_{1}, \ldots w_{r}\right\rangle$ is virtually geometric, then there are positive integers $k_{1}, \ldots, k_{r}$ so that

$$
\left\langle x_{1}, \ldots, x_{n} \mid w_{1}^{k_{1}}, \ldots w_{r}^{k_{r}}\right\rangle
$$

is virtually a $3-$ manifold group. Indeed, realizing $\underline{w}=\left\{w_{1}, \ldots, w_{r}\right\}$ as a collection of embedded loops $N_{\underline{w}}$ in a genus $n$ handlebody $H$, let $\tilde{H} \stackrel{p}{\longrightarrow} H$ be a regular finite cover in which the preimage $\pi^{-1}\left(N_{\underline{w}}\right)$ is homotopic to an embedding in the boundary. For $i \in\{1, \ldots, r\}$ let $\gamma_{i} \subset H$ be the loop in $N_{\underline{w}}$ corresponding to $w_{i}$. Each component of $p^{-1}\left(\gamma_{i}\right)$ covers $\gamma_{i}$ with the same degree; let $k_{i}$ be this degree. We construct a complex $\tilde{K}$ from $\tilde{H}$ by attaching $k_{i}$ disks to each component of $p^{-1}\left(\gamma_{i}\right)$, for each $i$. Since $p^{-1}\left(N_{\underline{w}}\right)$ is homotopic to an embedding into $\partial \tilde{H}$, the complex $\tilde{K}$ is homotopy equivalent to a 3 -manifold. Moreover, the covering map $\tilde{H} \rightarrow H$ extends to a covering map $\tilde{K} \rightarrow K$ where $K$ is a complex with $\pi_{1}(K)=\left\langle x_{1}, \ldots, x_{n} \mid w_{1}^{k_{1}}, \ldots w_{r}^{k_{r}}\right\rangle .^{1}$

\footnotetext{
${ }^{1}$ Actually, one can use the Orbifold Theorem $[2,7]$ together with a theorem of McCullough and Miller [15] to show that

$$
\left\langle x_{1}, \ldots, x_{n} \mid w_{1}^{N}, \ldots w_{r}^{N}\right\rangle
$$

is a virtual 3-manifold group for some positive integer $N$, but the author does not know an elementary argument.
} 
(An aside: It is possible to show that every finitely presented group admits a presentation which is virtually geometric, but the virtually geometric presentation will nearly always be different from the original one. Briefly, one realizes the group as the fundamental group of a special polyhedron; a finite branched cover of this polyhedron with positive even-degree branching over the center of every face will be thickenable. It follows that a presentation coming from the special polyhedron is virtually geometric. For more on special polyhedra and thickenability, see Chapter 1 of [14].)

The double $D_{n}(\underline{w})$ along a virtually geometric collection $\underline{w}$ is also virtually the fundamental group of a 3-manifold, obtained by joining together a pair of handlebodies by thickened annuli. A 3-manifold argument based on Dehn's Lemma [10, Lemma $20]$ then shows that if $D_{n}(\underline{w})$ is one-ended, it contains a closed surface subgroup.

Gordon and Wilton gave some examples of virtually geometric but non-geometric words in [10], and asked the following:

Question 1.6. [10, Question 22] Let $F$ be a free group of rank $n \geq 2$. Is $\{[w]\}$ virtually geometric for every $w \in F$ ?

We give a negative answer to this question in Section 4, by showing that the word bbaaccabc $\in\langle a, b, c\rangle$ is not virtually geometric (See Corollary 4.2).

Remark 1.7. Danny Calegari (personal communication) pointed out a second motivation for studying Question 1.6, connected with stable commutator length [4]. If a homologically trivial collection $\underline{w}$ is geometric, then $\operatorname{scl}(\underline{w})$ is in $\mathbb{Z}+\frac{1}{2}$, by $[4$, Proposition 4.4]. (This Proposition is an application of Gabai's result $[9,6.18]$ on the proportionality of Thurston and Gromov norms on the second homology of a 3-manifold.) More generally, suppose $\underline{w}$ is virtually geometric, so that $\underline{w}_{F^{\prime}}$ is geometric for some $F^{\prime}$ with $\left[F: F^{\prime}\right]<\infty$. A straightforward argument then shows that

$$
\operatorname{scl}(\underline{w}) \in \frac{1}{\left[F: F^{\prime}\right]}\left(\mathbb{Z}+\frac{1}{2}\right) .
$$

Thus a positive answer to Question 1.6 for words in $[F, F]$ would have given an alternate proof of the rationality of scl in free groups, which is the main theorem of [5]. In fact, the answer to Question 1.6 is negative even in this more restricted setting (see Corollary 4.3).

\section{Whitehead graphs}

This section describes certain graphs which arise naturally from collections of words together with disk systems in handlebodies. The material in this section is discussed in more detail in Berge [1].

In the graphs we consider, multiple edges may connect a pair of vertices, but no edge joins a vertex to itself. The valence of a vertex is the number of incident edges (not the number of neighbors), and a graph will be said to be regular if every vertex has the same valence.

A collection of conjugacy classes in a free group $F=\left\langle x_{1}, \ldots, x_{n}\right\rangle$ can also be thought of as a collection of cyclic words, i.e., freely and cyclically reduced "words" $w_{1}, \ldots, w_{r}$ whose letters are indexed $\bmod l_{i}$ where $l_{i}$ is the length of $w_{i}$. Given a collection $\underline{w}$ of cyclic words, one can form the Whitehead graph $W(\underline{w})$ as follows: 
(1) The $2 n$ vertices of $W(\underline{w})$ are the generators $x_{1}, \ldots, x_{n}$ of $F$ and their inverses.

(2) For each two-letter sequence $x y$ in some $w_{i} \in \underline{w}$, attach an edge from $x^{-1}$ to $y$. (If some $w_{i}=x$ has length one, attach an edge from $x^{-1}$ to $x$.)

Example 2.1. If $F$ is the free group $\langle a, b, c\rangle$, and $\underline{w}=\{b b a a c c a b c\}$, then the Whitehead graph $W(\underline{w})$ is isomorphic to the complete bipartite graph $K_{3,3}$.

Remark 2.2. Again, there is a topological interpretation: Realize the collection $\underline{w}$ as an embedded 1-submanifold $N$ in the handlebody $H$. There is a collection of disks $\mathcal{D}=\left\{d_{1}, \ldots, d_{n}\right\}$ in $H$ so that for each $i$ there is a loop in $H$ representing the generator $x_{i}$ which intersects $d_{i}$ exactly once, and has empty intersection with $d_{j}$ for all $j \neq i$. After homotoping $N$ to intersect $\mathcal{D}$ minimally, split the handlebody along $\mathcal{D}$ to give a ball with $2 n$ disks $\mathcal{D}_{ \pm}$in the boundary, and $N^{\prime}$ a 1 -submanifold with boundary in $\mathcal{D}_{ \pm}$. The Whitehead graph can then be obtained as the quotient of $\mathcal{D}_{ \pm} \cup N^{\prime}$ by crushing each disk to a point.

Notice that if $N$ was homotopic to an embedding into $\partial H$ which still intersects $\mathcal{D}$ minimally, then the Whitehead graph is planar. As shown by an example of Berge $[1$, Section 12], the assumption that $N$ still intersects $\mathcal{D}$ minimally after being homotoped into $\partial H$ is necessary, even for geometric $\underline{w}$; there are examples of geometric collections of words with non-planar Whitehead graph. However, we will see in Theorem 2.3 that if $\mathcal{D}$ is minimal in a certain sense, and $\underline{w}$ is geometric, then the Whitehead graph is planar.

A complete system of disks for $H$ is a collection $\mathcal{C}$ of properly embedded disks which is maximal with respect to the condition that $H \backslash \cup \mathcal{C}$ is connected. The system $\mathcal{D}$ from Remark 2.2 is an example of a complete system. Different choices of disks determine different choices of free generators for $\pi_{1}(H)$. (Actually, there are multiple complete disk systems corresponding to any choice of free generators; these disk systems are related to one another by homeomorphisms of $H$ which act trivially on $\pi_{1}(H)$.)

Any collection of disjoint properly embedded disks in $H$ (complete or not) gives rise to a graph $W(\underline{w}, \mathcal{C})$ in exactly the same manner as described in Remark 2.2. So long as $\mathcal{C}$ is a complete system, the graph $W(\underline{w}, \mathcal{C})$ always has $2 n$ vertices, but the number of edges depends on the particular complete system $\mathcal{C}$. (The number of edges is the number of times $\mathcal{C}$ intersects $\underline{w}$, after $\underline{w}$ has been homotoped to make this intersection minimal.) The complete system $\mathcal{C}$ is said to be minimal (with respect to $\underline{w})$ if the number of edges is minimal over all complete systems of disks for $H$.

As explained in [1], the following can be deduced from a result of Zieschang [6, Theorem 1].

Theorem 2.3. [1, Section 11] If $\mathcal{C}$ is minimal, and $\underline{w}$ is geometric, then $W(\underline{w}, \mathcal{C})$ is planar.

(In case the hypotheses of Theorem 2.3 are satisfied, Berge [1, Section 13] explains further how to obtain a Heegaard diagram, and thus an explicit embedding of $N_{\underline{w}}$ in $\partial H$, from $W(\underline{w}, \mathcal{C})$.

The next theorem is explained in [1, Section 8], and follows from the "peak reduction" lemma of Higgins-Lyndon (see [11] or [13, I.4], cf. [8, Section 4] for an account with a different flavor). 
Theorem 2.4. [1, Section 8] If $\mathcal{C}$ is not minimal, then $W(\underline{w}, \mathcal{C})$ contains a pair of vertices $v_{+}$and $v_{-}$with the same valence $k$ and a collection of $s<k$ edges $\left\{e_{1}, \ldots, e_{s}\right\}$ which separate $v_{+}$from $v_{-}$.

Remark 2.5. The pair of vertices $v_{ \pm}$in Theorem 2.4 is the pair of vertices coming from some single disk $D$ in $\mathcal{C}$. One can obtain a new disk system $\mathcal{C}^{\prime}$ for $H$ by deleting $D$ and adding a disk $D^{\prime}$ which intersects each of the edges $e_{1}, \ldots, e_{s}$ exactly once, without intersecting any other edges. The Whitehead graph $W\left(\underline{w}, \mathcal{C}^{\prime}\right)$ is obtained from $W(\underline{w}, \mathcal{C})$ by adding a new pair of vertices (corresponding to $D^{\prime}$ ), deleting $\left\{v_{ \pm}\right\}$, and "splicing together" the edges incident to $\left\{v_{ \pm}\right\}$. (We will give a precise definition of splicing in the next section.) An example of such an operation (called a Whitehead move) is shown in Figure 1.

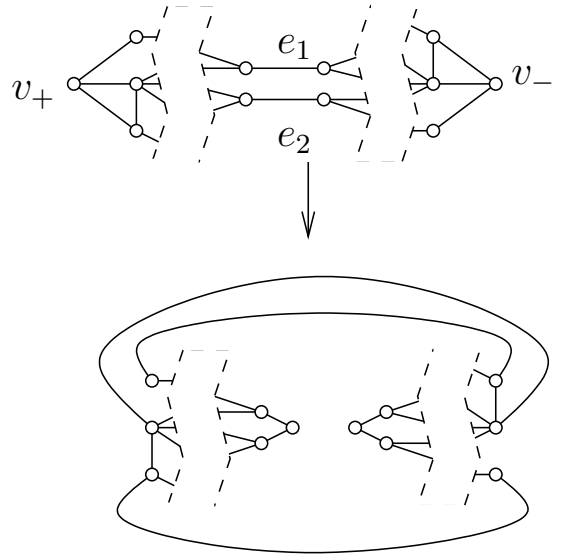

FiguRE 1. Reducing the number of edges in the Whitehead graph by a Whitehead move (an "unsplice" followed by a splice).

\section{Splicing}

Let $G_{1}$ and $G_{2}$ be graphs containing vertices $v_{1} \in G_{1}, v_{2} \in G_{2}$ of the same valence. Let $\sigma$ be a bijection from the edges incident to $v_{1}$ to the edges incident to $v_{2}$. We can form a new graph $G$ from this data by the procedure:

(1) Form the disjoint union of $G_{1}$ and $G_{2}$.

(2) Delete the vertices $v_{1}$ and $v_{2}$ (and any adjacent edges).

(3) For each edge $e$ connecting $v_{1}$ to some $v^{\prime} \in G_{1}$, the corresponding edge $\sigma(e)$ connects $v_{2}$ to some $v^{\prime \prime} \in G_{2}$. Add an edge to $G$ connecting $v^{\prime}$ to $v^{\prime \prime}$.

If $G$ is obtained by such a procedure, we say that $G$ is obtained by splicing $G_{1}$ and $G_{2}$ at $\left(v_{1}, v_{2}\right)$. (There may be more than one such $G$ for a given $G_{1}, G_{2},\left(v_{1}, v_{2}\right)$.)

Lemma 3.1. Let $v_{i} \in G_{i}$ be vertices for $i \in\{1,2\}$. If $G_{2} \backslash v_{2}$ is connected, and $G$ is obtained by splicing $G_{1}$ and $G_{2}$ at $\left(v_{1}, v_{2}\right)$, then $G_{1}$ is a minor of $G$.

Proof. Collapse $G_{2} \backslash v_{2}$ to a point to obtain a graph isomorphic to $G_{1}$. 
Definition 3.2. We say a graph is $p$-edge-connected if no collection of $p-1$ or fewer edges disconnects the graph.

Lemma 3.3. Let $k>0$, and suppose that $G$ is obtained by splicing $G_{1}$ and $G_{2}$ at $\left(v_{1}, v_{2}\right)$. If $G_{1}$ and $G_{2}$ are regular, $k$-valent, $k$-edge-connected graphs, then so is $G$.

Proof. For $i \in\{1,2\}$, let $G_{i}^{\prime}$ be the graph obtained from $G_{i}$ by removing $v_{i}$ and all the edges adjacent to $v_{i}$. The graph $G_{i}^{\prime}$ will be thought of either as a subgraph of $G_{i}$ or as a subgraph of $G$, depending on context.

For $i \in\{1,2\}$ we claim that $G_{i}^{\prime}$ is $\left(\left\lfloor\frac{k-1}{2}\right\rfloor+1\right)$-edge-connected. Indeed, suppose that $G_{1}^{\prime}$ is disconnected by $\left\lfloor\frac{k-1}{2}\right\rfloor$ or fewer edges into components $C_{1}, \ldots C_{c}$ for some $c \geq 2$. For some $1 \leq j \leq c$, the number of edges connecting $v_{1}$ to $C_{j}$ in $G_{1}$ is at most $\left\lfloor\frac{k}{2}\right\rfloor$. It follows that $G_{1}$ can be disconnected by at most $\left\lfloor\frac{k-1}{2}\right\rfloor+\left\lfloor\frac{k}{2}\right\rfloor=k-1$ edges, contradicting the $k$-edge-connectedness of $G_{1}$.

Let $E$ be a set of $(k-1)$ or fewer edges in $G$. Either $G_{1}^{\prime}$ or $G_{2}^{\prime}$ contains at most $\left\lfloor\frac{k-1}{2}\right\rfloor$ edges in $E$. Switching labels if necessary, we suppose it is $G_{1}^{\prime}$. Since $G_{1}^{\prime}$ is $\left(\left\lfloor\frac{k-1}{2}\right\rfloor+1\right)$-edge-connected, the subgraph $G_{1}^{\prime} \backslash E \subset G \backslash E$ is connected.

Let $x_{1}, \ldots, x_{k}$ be the (not necessarily distinct) vertices of $G_{1}$ connected to $v_{1}$, and let $E_{0}$ be the set of $k$ edges in $G$ connecting $G_{1}^{\prime}$ to $G_{2}^{\prime}$. Finally, let $G_{2}^{\prime \prime} \subset G$ be

$$
G_{2}^{\prime} \cup\left\{x_{1}, \ldots, x_{k}\right\} \cup E_{0} .
$$

Notice that there is a quotient map $p: G_{2}^{\prime \prime} \rightarrow G_{2}$ which takes each $x_{j}$ to $v_{2}$. Since $G_{2}$ is $k$-edge-connected, $p\left(E \cap G_{2}^{\prime \prime}\right)$ doesn't disconnect $G_{2}$. This implies that every vertex of $G_{2}^{\prime \prime}$ is connected in $G_{2}^{\prime \prime} \backslash E$ to $x_{j}$ for some $j$. Since the $x_{j}$ are all in $G_{1}^{\prime}$, and $G_{1}^{\prime} \backslash E$ is connected, $G \backslash E$ is connected.

We now explain how splicing arises in building Whitehead graphs. Let $\underline{w}$ be a collection of words, as in Section 2. Let $\mathcal{C}$ be a complete system of disks for the handlebody $H$, and let $\tilde{H}$ be some $d$-fold cover of $H$, with $\pi_{1}(\tilde{H})=F^{\prime}$. The preimage $\tilde{\mathcal{C}}$ in $\tilde{H}$ has too many disks to be a complete disk system for $\tilde{H}$, but we can still form the graph $W\left(\underline{w}_{F^{\prime}}, \tilde{\mathcal{C}}\right)$. Each component of the complement of $\tilde{\mathcal{C}}$ looks exactly like the complement of $\mathcal{C}$ in $H$, so we obtain the following:

Lemma 3.4. The graph $W\left(\underline{w}_{F^{\prime}}, \tilde{\mathcal{C}}\right)$ is isomorphic to a disjoint union of $d$ copies of $W(\underline{w}, \mathcal{C})$.

To get a complete system of disks for $\tilde{H}$, we must delete some disks from $\tilde{\mathcal{C}}$. Each time we delete one of these disks, the number of complementary components goes down by one, and the Whitehead graph changes by splicing two components together:

Lemma 3.5. Let $\mathcal{D}_{0} \subseteq \mathcal{D}_{1} \subset \mathcal{D}_{2}$ be disk systems in a handlebody $H$ so that $\mathcal{D}_{0}$ is complete and $\mathcal{D}_{2} \backslash \mathcal{D}_{1}$ is a single disk. Let $\underline{w}$ be a collection of conjugacy classes in $\pi_{1}(H)$. The graph $W_{1}=W\left(\underline{w}, \mathcal{D}_{1}\right)$ is obtained from $W_{2}=W\left(\underline{w}, \mathcal{D}_{2}\right)$ by splicing two components of $W_{2}$ together at a vertex.

(Similarly, the Whitehead move in Figure 1 can be described as an "unsplicing" of the edges $e_{1}, \ldots, e_{s}$ followed by splicing the resulting components together at $v_{ \pm}$.) 


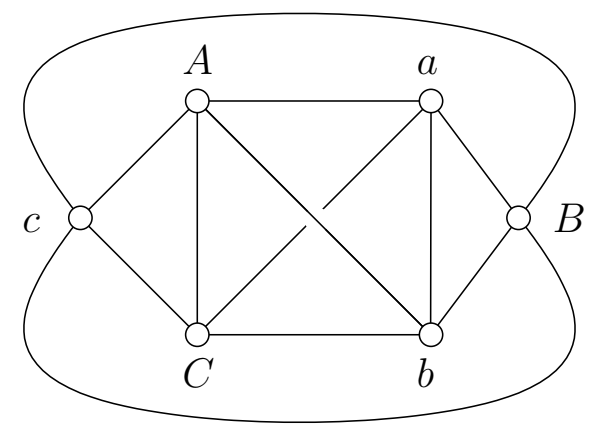

Figure 2. The Whitehead graph of the word $w=b a a b c c A C B B C A$ is non-planar, 4-valent, and 4-edge-connected.

\section{Examples}

Theorem 4.1. Let $\mathcal{C}$ be a complete system of disks for the handlebody $H$, and let $w$ be a collection of conjugacy classes in $\pi_{1}(H)$. Let $k \geq 3$. If $W(\underline{w}, \mathcal{C})$ is a regular, $k$-valent, $k$-edge-connected non-planar graph, then $\underline{w}$ is not virtually geometric.

Proof. Let $\tilde{H}$ be a $d$-fold cover of $H$, corresponding to $F^{\prime}<F$, where $F=\pi_{1}(H)$, and let $\tilde{\mathcal{C}}$ be the preimage of $\mathcal{C}$ in $\tilde{H}$.

By Lemma 3.4, the Whitehead graph $W\left(\underline{w}_{F^{\prime}}, \tilde{\mathcal{C}}\right)$ is equal to $d$ disjoint copies of $W(\underline{w}, \mathcal{C})$. If $\mathcal{D} \subset \tilde{\mathcal{C}}$ is a complete system of disks for $\tilde{H}$, then Lemma 3.5 implies that $W\left(\underline{w}_{F^{\prime}}, \mathcal{D}\right)$ is obtained from $W\left(\underline{w}_{F^{\prime}}, \tilde{\mathcal{C}}\right)$ by successively splicing together distinct components in some way (determined by the choice of complete system $\mathcal{D} \subset \tilde{\mathcal{C}}$ ) until only $2 \cdot \operatorname{rank}\left(F^{\prime}\right)$ vertices remain. Exactly $d-1$ splices will be performed, and each splice reduces the number of components by one, by Lemma 3.3 , so $W\left(\underline{w}_{F^{\prime}}, \mathcal{D}\right)$ will be connected.

Moreover, induction on $d$ together with Lemma 3.3 imply that $W\left(\underline{w}_{F^{\prime}}, \mathcal{D}\right)$ is $k-$ valent and $k$-edge-connected. By Theorem 2.4, the disk system $\mathcal{D}$ is minimal.

A $k$-valent, $k$-edge-connected graph cannot be disconnected by removing a single vertex, so induction on $d$ and Lemma 3.1 imply that the graph $W(\underline{w}, \mathcal{C})$ is a minor of $W\left(\underline{w}_{F^{\prime}}, \mathcal{D}\right)$. It follows that $W\left(\underline{w}_{F^{\prime}}, \mathcal{D}\right)$ is non-planar.

Theorem 2.3 then implies that $\underline{w}_{F^{\prime}}$ is not geometric in $\tilde{H}$. Since the finite cover $\tilde{H}$ was arbitrary, $\underline{w}$ is not virtually geometric.

A concrete example is the word bbaaccabc $\in F_{3}=\langle a, b, c\rangle$; the Whitehead graph $W(\{b b a a c c a b c\})$ is isomorphic to the complete bipartite graph $K_{3,3}$. This graph is 3 valent, 3-edge-connected, and non-planar. Applying Theorem 4.1 gives the following.

Corollary 4.2. The word $w=$ bbaaccabc in $F_{3}=\langle a, b, c\rangle$ is not virtually geometric.

For an example lying in the commutator subgroup, we take $w=$ baabccACBBCA. (We use the common convention $A=a^{-1}, B=b^{-1}, C=c^{-1}$.) The Whitehead graph, shown in Figure 2, is non-planar, 4-valent, and 4-edge-connected, so Theorem 4.1 immediately gives the following.

Corollary 4.3. The word $w=$ baabccACBBCA in $F_{3}=\langle a, b, c\rangle$ is not virtually geometric. 
The curious reader should be able to construct numerous similar examples, but it should be noted that either of the above examples gives non-virtually geometric words in $F_{n}$ for all $n \geq 2$, as the following proposition shows.

Proposition 4.4. Let $\underline{w}=\{w\}$ be a non-virtually geometric collection of words in $F_{3}$. For all $n \geq 2$ there is an embedding $\phi_{n}: F_{3} \rightarrow F_{n}$ so that $\phi_{n}(\underline{w})$ is not virtually geometric in $F_{n}$.

Proof. For $n=2$ we argue as follows: Let $\phi_{2}: F_{3} \rightarrow F_{2}$ be any map with image an index 2 subgroup. Indeed, the homomorphism $\phi_{2}$ is realized by a double cover of handlebodies $H_{3} \rightarrow H_{2}$, and any cover $\tilde{H}$ of $H_{2}$ either covers $H_{3}$ itself or has a double cover which covers $H_{3}$. The lifts of $\underline{w}$ in this cover are contained in the lifts of $\phi_{2}(\underline{w})$. Thus if $\phi_{2}(\underline{w})$ were virtually geometric, then $\underline{w}$ would be virtually geometric.

For $n>3$, we let $\phi_{n}: F_{3} \rightarrow F_{n}$ be any embedding of $F_{3}$ as a free factor of $F_{n}$. To see why $\phi_{n}(\underline{w})$ is non-virtually geometric, it is easiest to argue topologically. Let $H_{3}$ be the handlebody of genus 3 ; we can obtain a handlebody $H_{n}$ of genus $n$ by attaching $n-3$ one-handles along $2 n-6$ disjoint disks in the boundary of $H_{3}$. Realize $\underline{w}$ as an embedded 1-manifold $N$ in $H_{3} \subset H_{n}$. Suppose there is some finite cover $\tilde{H} \stackrel{\bar{p}}{\rightarrow} H_{n}$ in which the preimage of $N$ is homotopic to an embedded submanifold of the boundary. There is a corresponding finite cover $\tilde{H}^{\prime} \stackrel{p^{\prime}}{\rightarrow} H_{3}$ so $\tilde{H}^{\prime}$ embeds in $\tilde{H}$. There is also an infinite-sheeted cover $\tilde{H}^{\prime \prime} \stackrel{p^{\prime \prime}}{\rightarrow} \tilde{H}$ corresponding to $\pi_{1}\left(\tilde{H}^{\prime}\right) \subset \pi_{1}(\tilde{H})$; the space $\tilde{H}^{\prime \prime}$ can be obtained from $\tilde{H}^{\prime}$ by attaching finitely many non-compact 3-manifolds with boundary $A_{1}, \ldots, A_{l}$ to $\tilde{H}^{\prime}$ along disks. Each of the $A_{i}$ is homeomorphic to a ball with a Cantor set removed from its boundary.

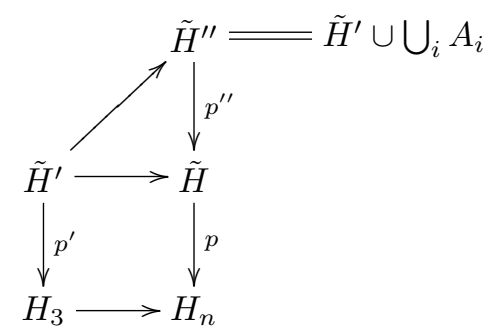

Notice that $p^{-1}(N) \subseteq\left(p \circ p^{\prime \prime}\right)^{-1}(N)$ coincides with the union of the compact components of $\left(p \circ p^{\prime \prime}\right)^{-1}(N)$. If $p^{-1}(N)$ is homotopic to an embedding in the boundary of $\tilde{H}$, then $\left(p \circ p^{\prime \prime}\right)^{-1}(N)$ is homotopic to an embedding in the boundary of $\tilde{H}^{\prime \prime}$. Since $\tilde{H}^{\prime \prime}=\tilde{H}^{\prime} \cup \bigcup_{i} A_{i}$, this embedding is homotopic to an embedding in the boundary of $\tilde{H}^{\prime}$, which covers $H_{3}$. This contradicts the assumption that $\underline{w}$ is not virtually geometric in $F_{3}$.

Remark 4.5. Gordon and Wilton's motivation for asking Question 1.6 was the question of which doubles of free groups contain closed surface subgroups. The double $D_{3}(\{b a a b c c A C B B C A\})$ contains a surface subgroup, by an application of the main theorem of $[3]$. The double $D_{3}(\{b b a a c c a b c\})$ contains a closed surface subgroup by an explicit construction of Sang-hyun Kim [12]. 


\section{Acknowledgments}

The author thanks Danny Calegari for pointing out this problem, Danny Calegari, Daryl Cooper, and Richard Schwartz for useful conversations, and Nathan Dunfield for a python script which was useful for running computer experiments with John Berge's program Heegaard. Thanks also to John Berge for pointing out a bad typo in an earlier version of this note, and thanks to the anonymous referee for useful comments.

The author was visiting the Caltech mathematics department while this work was done, and thanks Caltech for their hospitality. This work was partly supported by the National Science Foundation, grant DMS-0804369.

\section{References}

[1] J. Berge, Documentation for the program Heegaard. Preprint (c.1990), available at http://www . math. uic.edu/ $\sim 3 m$.

[2] M. Boileau, B. Leeb, and J. Porti, Geometrization of 3-dimensional orbifolds, Ann. of Math. (2) 162 (2005), no. 1, 195-290.

[3] D. Calegari, Surface subgroups from homology, Geom. Topol. 12 (2008), no. 4, 1995-2007.

[4] - scl, Vol. 20 of MSJ Memoirs, Mathematical Society of Japan, Tokyo (2009), ISBN 978-4-931469-53-2.

[5] - Stable commutator length is rational in free groups, J. Amer. Math. Soc. 22 (2009) 941-961.

[6] H. Cišang, Simple path systems on full pretzels, Mat. Sb. (N.S.) 66 (108) (1965) 230-239. Translated in AMS Translations (2), vol. 92, pp. 127-137.

[7] D. Cooper, C. D. Hodgson, and S. P. Kerckhoff, Three-dimensional orbifolds and cone-manifolds, Vol. 5 of MSJ Memoirs, Mathematical Society of Japan, Tokyo (2000), ISBN 4-931469-05-1. With a postface by Sadayoshi Kojima.

[8] M. Culler and K. Vogtmann, Moduli of graphs and automorphisms of free groups, Invent. Math. 84 (1986), no. 1, 91-119.

[9] D. Gabai, Foliations and the topology of 3-manifolds, J. Differential Geom. 18 (1983), no. 3, 445-503.

[10] C. Gordon and H. Wilton, On surface subgroups of doubles of free groups. Preprint, arXiv: 0902.3693v1.

[11] P. J. Higgins and R. C. Lyndon, Equivalence of elements under automorphisms of a free group, J. London Math. Soc. (2) 8 (1974) 254-258.

[12] S.-H. Kim, Geometricity and polygonality in free groups. Preprint, arXiv:0910.5019.

[13] R. C. Lyndon and P. E. Schupp, Combinatorial group theory, Springer-Verlag, Berlin (1977), ISBN 3-540-07642-5. Ergebnisse der Mathematik und ihrer Grenzgebiete, Band 89.

[14] S. Matveev, Algorithmic topology and classification of 3-manifolds, Vol. 9 of Algorithms and Computation in Mathematics, Springer-Verlag, Berlin (2003), ISBN 3-540-44171-9.

[15] D. McCullough and A. Miller, Manifold covers of 3-orbifolds with geometric pieces, Topology Appl. 31 (1989), no. 2, 169-185.

Department of Mathematics, University at Buffalo, SUnY, Buffalo, NY 14260

E-mail address: j399m@buffalo.edu 